\title{
Role of induction and adjuvant systemic treatment in mesothelioma: narrative review
}

\author{
Martin J. Edelman \\ Fox Chase Cancer Center, Philadelphia, PA, USA \\ Correspondence to: Martin J. Edelman, MD, FACP. Fox Chase Cancer Center, 333 Cottman Avenue, Philadelphia, PA 19111, USA. \\ Email: Martin.edelman@fccc.edu.
}

\begin{abstract}
Objective: This review will focus on the issues of integrating chemotherapy and immunotherapy in the definitive management of mesothelioma as either induction or adjuvant therapy.

Background: While surgery is considered a primary treatment for mesothelioma, complete resection can rarely be obtained. Systemic therapy with chemotherapy and more recently, immunotherapy has been demonstrated to improve outcomes in advanced disease. Therefore, it is appropriate to consider the use of chemotherapy and/or immunotherapy as induction therapy or postoperatively. Additionally, factors such as surgical procedure, radiotherapy and various prognostic factors also need to be considered.

Methods: A search of the English language literature was performed to identify prospective trials using current chemotherapy and immunotherapy regimens. In addition, literature was selectively reviewed for case series and database analyses that focused on the issue of the utility of induction or adjuvant therapy.

Conclusions: There are numerous issues that complicate the interpretation of studies, including the variety of surgical procedures, prognostic factors and the nature of the trial design and reporting. Relatively few well designed studies have been performed and there is inadequate data at this time to support or refute the use of induction or adjuvant therapy. Current studies are focused on evaluating immunotherapy studies in this setting.
\end{abstract}

Keywords: Chemotherapy; pemetrexed; immunotherapy; adjuvant

Received: 29 June 2021; Accepted: 20 August 2021; Published: 30 June 2022.

doi: 10.21037/asj-21-55

View this article at: https://dx.doi.org/10.21037/asj-21-55

\section{Introduction}

Pleural mesothelioma is an uncommon malignancy that is rarely amenable to definitive resection. Local and regional recurrence are common and the median overall survival is approximately 1 year (1). Therefore, multimodality therapy employing systemic therapies before or after resection, usually with radiotherapy as well, has been evaluated in attempts to improve outcome. The purpose of this review is to discuss issues in induction and adjuvant therapy including the drugs, issues in trial design and evaluation, the results of recent studies as well as the trials that are currently in progress. The following article is presented in accordance with the Narrative Review reporting checklist (available at https://asj.amegroups. com/article/view/10.21037/asj-21-55/rc).

\section{Methods}

Using PubMed, the English language literature was searched using the terms "mesothelioma", "adjuvant", "induction" and "neoadjuvant". In addition, article bibliographies and prior reviews were evaluated. Only studies conducted after 2003 were considered, as that was the time that the known effective regimens of platinum/ pemetrexed and later immunotherapy combinations were introduced. In addition, the clinicaltrials.gov data base was evaluated with the search terms "mesothelioma", "adjuvant" and "neoadjuvant" as well as the specific drug names to identify ongoing trials. Other papers that illustrated specific issues of interest in terms of historical issues and prognostic factors were also identified. 


\section{Systemic therapy}

Any discussion of induction or adjuvant systemic therapy must begin with a review of the known active regimens for the treatment of unresectable or advanced disease. The primary regimen for the treatment of mesothelioma is the combination of cisplatin/pemetrexed on the basis of an industry sponsored 574 patient randomized trial comparing the regimen to cisplatin alone published in 2003. The study demonstrated an advantage in terms of response $(41.3 \% \mathrm{vs}$. 16.7\%), progression free survival (PFS, 5.7 vs. 3.9 months) and overall survival (OS, 12.1 vs. 9.3 months) (2). A study employing a related anti-folate agent raltitrexed reached similar results, though did not lead to a U.S. Food and Drug Administration approval (3). The addition of the anti-vascular endothelial growth factor (VEGF) antibody bevacizumab to platinum/pemetrexed resulted in an improvement in all outcomes. Of note, the control group performed exceptionally well and it is likely that the overall study population was highly selected and not representative of the typical mesothelioma patient. United States FDA approval was never obtained and the combination was not widely adopted. Carboplatin, which has less renal toxicity, emetogenicity and neurotoxicity than cisplatin and does not require saline hydration (problematic in patients with cardiovascular disease) is frequently employed in lieu of cisplatin. It does have more myelotoxicity. Trials of carboplatin/pemetrexed have demonstrated comparable levels of activity (4). Evaluation of other cytotoxic chemotherapy agents (e.g., gemcitabine, vinorelbine etc.) demonstrated some activity in small phase II trials, but none was evaluated for activity in untreated patients in an adequately powered randomized study.

Studies of immunotherapy in mesothelioma began with the investigation of the anti-CTLA-4 agent tremelimumab. Initial reports appeared to demonstrate a high level of activity, which resulted in a rapidly accruing phase III trial (5). Despite two phase II trials demonstrating a promising level of activity, no benefit was observed and toxicity was increased in the phase III setting. In contrast, a phase III study $(\mathrm{n}=713)$ of the anti-CTLA-4 agent ipilimumab and anti-PD-1 drug nivolumab demonstrated substantial benefit in terms of overall survival (18.1 vs. 14.1 months, HR =0.74) vs. chemotherapy (platinum/ pemetrexed). PFS was similar but the duration of response for immunotherapy was substantially longer (11.0 vs. 6.7 months) (6). Remarkably, the greatest degree of benefit accrued to the non-epithelioid subtype, with superiority of immunotherapy over chemotherapy, while immunotherapy was at least comparable to chemotherapy in the patients with epithelioid disease.

\section{Considerations in evaluating therapy}

A major problem with assessing the utility of any of the available data regarding induction or adjuvant therapy for mesothelioma is that there are several confounding issues. Prognostic factors for chemotherapy were described in the 1990's for advanced disease by the Cancer and Leukemia Group B (7). This analysis evaluated patients entered onto prospective multicenter clinical trials of chemotherapy in the pre-pemetrexed era and found that Eastern Cooperative Group performance status, age, hemoglobin, white blood cell count, weight loss and chest pain were major prognostic factors. Others have noted mesothelioma histology as a major factor with the epithelioid variant responding better and having superior survival over mixed or sarcomatoid type disease. The European Organization for the Research and Treatment of Cancer (EORTC) performed a similar analysis with similar results finding that performance status, white blood cell count, male sex were prognostic factors (8).

In the neoadjuvant/adjuvant setting there are additional considerations. First, there is considerable variability in the nature of the surgical procedure. Surgery for mesothelioma (discussed elsewhere) is uniquely different than most other cancer surgeries in that an R0 resection is usually not feasible (8). Two major approaches, lung sacrificing extrapleural pneumonectomy (EPP) and lung sparing pleurectomy/decortication $(\mathrm{P} / \mathrm{D})$ have been employed. Therefore, post-operative treatment is not "adjuvant" therapy in the usual sense in that there is almost always known residual disease. While there is still considerable disagreement, an emerging consensus is that $\mathrm{P} / \mathrm{D}$ represents an optimal approach, though there is an absence of a clear definition of this procedure $(9,10)$. For any pleural mesothelioma surgery the goal is to achieve a macroscopic complete resection (R1) — typically defined as no residual palpable or visible disease. A prognostic score was developed based upon a retrospective review of patients who were considered candidates for chemotherapy followed by surgery with extrapleural pneumonectomy (EPP) in the "modern era" of chemotherapy (regimen was either cisplatin/gemcitabine or cisplatin/pemetrexed) (11). In this study, response to chemotherapy emerged as a major prognostic factor.

Reporting of data from various studies is variable, with 
survivals frequently calculated from different time points, including diagnosis, date of initiation of systemic therapy, date of surgery etc. This makes the already difficult task of cross trial outcome assessment even more difficult. To date, there are no conclusive data regarding the utility of either approach. Another important aspect to remember is that most trials also plan for patients to receive radiotherapy, adding another variable. Therefore, there are at least three major therapeutic variables to consider when evaluating trials: the chemotherapy regimen, surgical procedure and radiation therapy and technique. All of these have evolved significantly over time. This evolution has not only included obvious changes (e.g., differences in drugs or surgical procedure), but also subtle issues (radiation technique, e.g., 3-D conformal planning to intensity modulated radio therapy; improved supportive care for chemotherapy; adoption of CT/PET for staging etc.) that can improve outcomes without seeming to change the actual treatment strategy.

The appropriate outcome for such studies is also subject to debate. It is typical for oncology studies to report response data, PFS and OS. The former is difficult to assess in mesothelioma radiologically, though pathologic data from resected specimens is quite clear. Complete pathologic response, when reported, is usually a marker of good prognosis. However, such responses are infrequent and not every patient who commences multidisciplinary therapy will undergo resection. PFS and OS are reported as medians, which are clearly relevant but do not tell the entire story. A treatment that is highly toxic may have early morbidity and mortality which may obscure the durable effect (i.e., cure) in a subset of patients. This subset will be the "tail of the curve" on a Kaplan-Meir plot. Given the nature of the disease, this subset, i.e., the proportion of durable responders, is probably the most relevant outcome to consider.

There have been recent comprehensive reviews of multimodality therapy for mesothelioma. For the purposes of this chapter, the focus will be on prospective studies employing modern regimens that use approved drugs (i.e., platinum/pemetrexed or immunotherapy). Intracavitary therapy will not be discussed.

\section{Induction or adjuvant}

The timing of systemic therapy in potentially resectable solid tumors is controversial. Induction therapy allows for assessment of the actual responsiveness of the tumor as well as for the ability to obtain substantial tissue for further evaluation. Treatment can be instituted rapidly and patients are better able to tolerate side effects as they are not recovering from a major surgery. It is possible that induction therapy could lead to response and permit a more complete resection (down staging). A disadvantage is the delay of the "definitive" procedure as well as the potential that treatment toxicity could result in permanent deferral of surgery. Post-operative or adjuvant therapy has the advantage that the bulk of tumor has been removed and therefore less likelihood that inherently resistant clones are present. A major disadvantage is that patients are usually less fit and frequently less able to tolerate the toxicity of currently available agents.

An evaluation of the National Cancer Data Base of induction and adjuvant chemotherapy found that the overall outcomes appear similar, though postoperative outcomes were inferior for those who received induction therapy (12). A problem with an analysis of this type is that it cannot assess patients who never underwent surgery due to toxicity nor determine how many patients who initially were not considered for surgery ultimately received an operation after a favorable response to treatment. A randomized phase II comparison of pre- $v s$. post-operative therapy is currently underway (EORTC 1205). This trial employs pleurectomy/ decortication as the operative procedure and uses platinum/ pemetrexed as the systemic therapy (13).

\section{Induction therapy}

Despite a number of trials, there is an absence of clear data supporting multimodality therapy in mesothelioma patients who are surgical candidates. No randomized trial has been performed. Furthermore, all trials of multimodality therapy to date have employed chemotherapy. Studies done before the development of pemetrexed primarily employed platinum/gemcitabine, a regimen that lacks a clear demonstration of improved outcomes in advanced disease (14). Most recent studies (i.e., after 2005) usually employ the platinum/pemetrexed regimen. Given the rapid developments with immunotherapy and exciting preliminary data of chemoimmunotherapy and immunotherapy as induction therapy for non-small cell lung cancer, it is only a matter of time before this approach is explored in mesothelioma.

A number of small prospective studies have been performed evaluating the induction strategy. Table 1 presents selected recent studies that employed the platinum/pemetrexed regimen. Krug led the first of these studies and demonstrated the feasibility of the approach. 
Table 1 Prospective studies using induction platinum/pemetrexed for pleural mesothelioma

\begin{tabular}{|c|c|c|c|c|c|c|c|c|c|}
\hline Study & Year & Number & $\begin{array}{c}\mathrm{N} \\
\text { cycles }\end{array}$ & $\begin{array}{l}\text { Surgical } \\
\text { procedure }\end{array}$ & $\begin{array}{l}\text { N completing } \\
\text { surgery }\end{array}$ & XRT & $\begin{array}{c}\text { N completing all } \\
\text { therapy }\end{array}$ & $\begin{array}{c}\text { PFS } \\
\text { (months) }\end{array}$ & $\begin{array}{c}\text { OS } \\
\text { (months) }\end{array}$ \\
\hline Krug (MSKCC) & 2009 & 77 & 4 & EPP & 54 & $\begin{array}{c}\text { Hemithoracic } \\
54 \text { Gy }\end{array}$ & $40 / 44$ & 10.1 & 16.8 \\
\hline Van Schil (EORTC) & 2010 & 57 & 3 & EPP & 46 & - & 24 & 13.9 & 18.4 \\
\hline Hasegawa (Japan MIG) & 2016 & 42 & 3 & EPP & 33 & - & 17 & 11 & 19.9 \\
\hline
\end{tabular}

EPP, extrapleural pneumonectomy; XRT, radiation therapy; PFS, progression free survival; OS, overall survival.

Patients with stage I-III disease, excellent PS, normal organ function etc. were eligible (15). Patients who had not progressed went on to surgery (EPP), and if resectable, hemithoracic radiotherapy to $54 \mathrm{~Gy}$. The primary endpoint was pathologic complete response, which was achieved in three patients. PFS and OS were 10.1 and 16.8 months, respectively. Patients who had radiologic, pathologic response, and completed all therapy had better outcomes. This trial exemplifies a number of issues with multimodality therapy of mesothelioma: small trial size, relatively few patients who are able to complete all planned therapy (40/77, 52\%) and better outcomes for those who respond to chemotherapy.

Trials employing a similar approach was undertaken by the EORTC (study 08031) and the Japan Mesothelioma Interest Group (study 0601) (16,17). The EORTC study enrolled 58 eligible patients. Similar to the MSKCC experience, only a minority of patients (37 of 58,64\%) completed therapy. The Japanese study enrolled 42 patients. Again, there was substantial drop off of patients at every point, only 30 underwent surgery and $17(40 \%)$ completed all planned therapy. OS was 19.9 months for the intention to treat population.

One of the largest induction trials actually questioned the use of surgery rather than the value of chemotherapy. The mesothelioma and radical surgery (MARS) trial evaluated EPP vs. radiation therapy after platinumbased chemotherapy (18). This study did not fully accrue with only 50 patients actually randomized. The major finding was that EPP resulted in unacceptable toxicity. Overall survival between the EPP and no EPP groups was 14.4 months vs. 19.5 months (HR $=1.90,95 \%$ CI: 0.92-3.93; exact $\mathrm{P}=0.082$ ), and after adjustment for sex, histological subtype, stage, and age at randomization the HR was 2.75 (95\% CI: 1.21-6.26; $\mathrm{P}=0.016$ ).

Another question raised by the studies described above is the value of radiotherapy. Stahel and the SAKK group evaluated the role of hemithoracic radiotherapy after neoadjuvant chemotherapy using cisplatin/pemetrexed followed by EPP (19). In a two-part, phase II trial patients were first enrolled induction chemotherapy with three cycles of cisplatin/pemetrexed, followed by EPP. Patients were then randomized to hemithoracic radiotherapy or observation. This trial enrolled a total of 151 patients, of whom 54 were ultimately randomized to radiotherapy $v s$. observation, with 27 randomized to radiotherapy and 25 actually completing radiation. No significant difference was found between the groups (median OS from randomization: $20.8 v s .19 .3$ months for observation $v s$. radiotherapy) with greater toxicity observed in the radiotherapy group. Therefore, at this time, post-operative radiotherapy is usually neither feasible nor effective in the post-operative setting.

An analysis of the National Cancer Data Base $(n=1,949)$ as well as the Duke University database $(\mathrm{n}=257)$ in 2020 failed to demonstrate any benefit from induction therapy (20). In fact, it reached the opposite conclusion, demonstrating that induction therapy actually increased post-resection mortality ( $\mathrm{HR}=1.29$ for the NCDB data, $\mathrm{HR}=1.62$, for propensity matched data).

\section{Adjuvant treatment}

Adjuvant treatment, i.e., administration of drug after resection when there is no known disease after an $\mathrm{R} 0$ resection, is the standard approach for preventing recurrence in a range of solid tumors including breast, lung, colon cancers. As noted above, mesothelioma represents a unique challenge in that R0 resection is difficult to clearly achieve and document. There are very few prospective studies of post-operative therapy and none that employs a platinum pemetrexed regimen. A number of retrospective studies have 
Table 2 Ongoing studies of immunotherapy as neoadjuvant/adjuvant therapy

\begin{tabular}{|c|c|c|c|c|c|c|c|}
\hline Lead organization/sponsor & NCT\# & Phase & $\begin{array}{l}\text { Planned } \\
\text { accrual }\end{array}$ & Agent(s) & $\begin{array}{l}\text { Surgical } \\
\text { procedure }\end{array}$ & \multicolumn{2}{|c|}{$\begin{array}{l}\text { Timing of immunotherapy } \\
\text { or chemoimmunotherapy } \\
\text { relative to surgery }\end{array}$} \\
\hline MSKCC & 04162015 & ॥ & 35 & Nivo + pem $/$ cis & $P / D$ & $x$ & \\
\hline Baylor & 02592551 & ॥ & 20 & Durva; Durva + Tremi & EPP or PD & $x$ & \\
\hline University of Chicago & 02707666 & ॥ & 15 & Pembro pre, pem/cis post & $P / D$ & $x$ & $x$ \\
\hline $\begin{array}{l}\text { Institut für Klinische } \\
\text { Krebsforschung IKF GmbH } \\
\text { at Krankenhaus Nordwest }\end{array}$ & 04177953 & $\begin{array}{l}\text { Randomized } \\
\text { phase II }\end{array}$ & 92 & $\begin{array}{l}\text { Nivo + pem/plat vs. pem/plat (post- } \\
\text { op) }\end{array}$ & $\begin{array}{l}\text { Extended } \\
\text { P/D }\end{array}$ & & $x$ \\
\hline $\begin{array}{l}\text { Southwest Oncology } \\
\text { Group }\end{array}$ & 03228537 & I & 28 & Atezo + cis/pem +/- XRT & PD or EPP & $x$ & $x$ \\
\hline
\end{tabular}

Nivo, nivolumab; Durva, durvalamab; Tremi, tremelimumab; pembro, pembrolizumab; pem, pemetrexed; cis, cisplatin; plat, cisplatin or carboplatin; atezo, atezolizumab; EPP, extrapleural pneumonectomy; P/D, pleurectomy/decortication; XRT, radiation therapy.

been published and recently reviewed (21). A typical report is the study by Bolukbas. This report evaluated 88 patients who were treated in a standardized manner that employed "radical pleurectomy" (a term that included P/D and EPP) followed by cisplatin/pemetrexed and then radiotherapy (22). These patients were selected from 206 that were evaluated by the multimodality team. Of the 88 patients, 83 were able to receive adjuvant platinum/pemetrexed therapy (usually with cisplatin). Patients received a median of four cycles of chemotherapy (range, 0-6). This experience does demonstrate that patients can receive chemotherapy in this setting, but does not establish efficacy.

The Mesothelioma and Radical Surgery 2 trial (MARS-2) is an ongoing study in the United Kingdom that evaluates the role of surgery (P/D) followed by chemotherapy with chemotherapy alone. This is an unusual and challenging trial design that recognizes the uncertain role of surgery in this disease (23). The planned enrollment for the trial is 328 , which will make it by far the largest prospective study of multimodality therapy in this disease. The study is scheduled to report primary findings at the end of 2022 .

\section{Other adjuvant/neoadjuvant approaches}

Logically building on the encouraging results of immunotherapy in advanced disease, a number of studies have commenced exploring the use of immunotherapy in potentially resectable disease (Table 2). Other novel agents, such as mesothelin directed antibody drug conjugates, have demonstrated activity in advanced disease (24). If confirmed, then evaluation in disease amenable to definitive therapy would be appropriate. An unexplored area would be to select agents based upon tumor characteristics (histologic, molecular etc.). For example, there are data that BAP1 mutation sensitizes to immunotherapy while resulting in resistance to gemcitabine $(25,26)$.

A number of other therapeutics in development have demonstrated activity in advanced disease and therefore, are reasonable to assess as part of a multimodality approach. Arginine deprivation represents a potentially targeted therapy for mesothelioma. Arginine is essential for biosynthesis of proteins, nitric oxide, and polyamines and contributes to proline and glutamate production. Arginosuccinate synthetase 1, an enzyme necessary for arginine synthesis is frequently (approximately $50 \%$, by immunohistochemistry) deficient in pleural mesothelioma. Normal tissues can dispense with exogenous arginine while deficient tumors rely on ASS-1. Therefore, depletion of exogenous arginine is a rational strategy. A randomized, multicenter phase II trial evaluating pegylated arginine deiminase in patients with ASS-1 deficient 
disease demonstrated improved PFS compared with best supportive care (3.2 vs. 2.0 months) in a randomized, phase II multicenter trial (27). This modest level of activity is not sufficient to advance the drug to the definitive setting, but combination with platinum/pemetrexed has been demonstrated to be feasible and the three-drug regimen may be appropriate (28). A trial of arginine deprivation in potentially resectable disease (NCT02709512) is ongoing.

\section{Conclusions}

Adjuvant and neoadjuvant therapy for localized mesothelioma is a logical approach and currently endorsed by treatment guidelines [e.g., National Comprehensive Cancer Network (NCCN), American Society of Clinical Oncology (ASCO)] despite the lack of clear randomized data demonstrating efficacy $(29,30)$. There has been considerable variability in approach and reporting that makes an objective selection of therapy difficult. Recent developments in systemic agents, particularly immunotherapy, hold substantial promise for improving outcomes at all stages.

\section{Acknowledgments}

Funding: This work was partially funded by P30 CA006927 from the National Cancer Institute.

\section{Footnote}

Provenance and Peer Review: This article was commissioned by the Guest Editors (Abbas E. Abbas and Stacey Su) for the series "Mesothelioma" published in AME Surgical fournal. The article has undergone external peer review.

Reporting Checklist: The author has completed the Narrative Review reporting checklist. Available at https://asj. amegroups.com/article/view/10.21037/asj-21-55/rc

Peer Review File: Available at https://asj.amegroups.com/ article/view/10.21037/asj-21-55/prf

Conflicts of Interest: The author has completed the ICMJE uniform disclosure form (available at https://asj.amegroups. com/article/view/10.21037/asj-21-55/coif). The series "Mesothelioma" was commissioned by the editorial office without any funding or sponsorship. The author reported the followings: Grants and contracts to his institution for clinical research from GSK, Windmil, Nektar, Revolutions Medicine and Adaptimmune; DSMB membership for trials sponsored by Astra Zeneca, Takeda, Seattle Genetics; Consulting fees from Windmil, Kanph, Sanofi; Lecture fees from Sanofi; Reimbursement for travel from Nektar; Deputy Editor for "Lung Cancer" and Chair of the Scientific Advisory Board for the Lung Cancer Foundation of America. The author has no other conflicts of interest to declare.

Ethical Statement: The author is accountable for all aspects of the work in ensuring that questions related to the accuracy or integrity of any part of the work are appropriately investigated and resolved.

Open Access Statement: This is an Open Access article distributed in accordance with the Creative Commons Attribution-NonCommercial-NoDerivs 4.0 International License (CC BY-NC-ND 4.0), which permits the noncommercial replication and distribution of the article with the strict proviso that no changes or edits are made and the original work is properly cited (including links to both the formal publication through the relevant DOI and the license). See: https://creativecommons.org/licenses/by-nc-nd/4.0/.

\section{References}

1. Mazurek JM, Syamlal G, Wood JM, et al. Malignant Mesothelioma Mortality - United States, 1999-2015. MMWR Morb Mortal Wkly Rep 2017;66:214-8.

2. Vogelzang NJ, Rusthoven JJ, Symanowski J, et al. Phase III study of pemetrexed in combination with cisplatin versus cisplatin alone in patients with malignant pleural mesothelioma. J Clin Oncol 2003;21:2636-44.

3. van Meerbeeck JP, Gaafar R, Manegold C, et al. Randomized phase III study of cisplatin with or without raltitrexed in patients with malignant pleural mesothelioma: an intergroup study of the European Organisation for Research and Treatment of Cancer Lung Cancer Group and the National Cancer Institute of Canada. J Clin Oncol 2005;23:6881-9.

4. Ceresoli GL, Zucali PA, Favaretto AG, et al. Phase II study of pemetrexed plus carboplatin in malignant pleural mesothelioma. J Clin Oncol 2006;24:1443-8.

5. Maio M, Scherpereel A, Calabrò L, et al. Tremelimumab as second-line or third-line treatment in relapsed malignant mesothelioma (DETERMINE): a multicentre, international, randomised, double-blind, placebo- 
controlled phase 2b trial. Lancet Oncol 2017;18:1261-73.

6. Baas P, Scherpereel A, Nowak AK, et al. First-line nivolumab plus ipilimumab in unresectable malignant pleural mesothelioma (CheckMate 743): a multicentre, randomised, open-label, phase 3 trial. Lancet 2021;397:375-86.

7. Herndon JE, Green MR, Chahinian AP, et al. Factors predictive of survival among 337 patients with mesothelioma treated between 1984 and 1994 by the Cancer and Leukemia Group B. Chest 1998;113:723-31.

8. Kantor T, Wakeam E. Landmark Trials in the Surgical Management of Mesothelioma. Ann Surg Oncol 2021;28:2037-47.

9. Zhou N, Rice DC, Tsao AS, et al. Extrapleural Pneumonectomy versus Pleurectomy/Decortication for Malignant Pleural Mesothelioma. Ann Thorac Surg 2022;113:200-8.

10. Flores RM, Pass HI, Seshan VE, et al. Extrapleural pneumonectomy versus pleurectomy/decortication in the surgical management of malignant pleural mesothelioma: results in 663 patients. J Thorac Cardiovasc Surg 2008;135:620-6, 626.e1-3.

11. Opitz I, Friess M, Kestenholz P, et al. A New Prognostic Score Supporting Treatment Allocation for Multimodality Therapy for Malignant Pleural Mesothelioma: A Review of 12 Years' Experience. J Thorac Oncol 2015;10:1634-41.

12. Verma V, Ahern CA, Berlind CG, et al. Treatment of malignant pleural mesothelioma with chemotherapy preceding versus after surgical resection. J Thorac Cardiovasc Surg 2019;157:758-66.e1.

13. Raskin J, Surmont V, Cornelissen R, et al. A randomized phase II study of pleurectomy/decortication preceded or followed by (neo-)adjuvant chemotherapy in patients with early stage malignant pleural mesothelioma (EORTC 1205). Transl Lung Cancer Res 2018;7:593-8.

14. Kindler HL, van Meerbeeck JP. The role of gemcitabine in the treatment of malignant mesothelioma. Semin Oncol 2002;29:70-6.

15. Krug LM, Pass HI, Rusch VW, et al. Multicenter phase II trial of neoadjuvant pemetrexed plus cisplatin followed by extrapleural pneumonectomy and radiation for malignant pleural mesothelioma. J Clin Oncol 2009;27:3007-13.

16. Van Schil PE, Baas P, Gaafar R, et al. Trimodality therapy for malignant pleural mesothelioma: results from an EORTC phase II multicentre trial. Eur Respir J 2010;36:1362-9.

17. Hasegawa S, Okada M, Tanaka F, et al. Trimodality strategy for treating malignant pleural mesothelioma: results of a feasibility study of induction pemetrexed plus cisplatin followed by extrapleural pneumonectomy and postoperative hemithoracic radiation (Japan Mesothelioma Interest Group 0601 Trial). Int J Clin Oncol 2016;21:523-30.

18. Treasure T, Lang-Lazdunski L, Waller D, et al. Extrapleural pneumonectomy versus no extra-pleural pneumonectomy for patients with malignant pleural mesothelioma: clinical outcomes of the Mesothelioma and Radical Surgery (MARS) randomised feasibility study. Lancet Oncol 2011;12:763-72.

19. Stahel RA, Riesterer O, Xyrafas A, et al. Neoadjuvant chemotherapy and extrapleural pneumonectomy of malignant pleural mesothelioma with or without hemithoracic radiotherapy (SAKK 17/04): a randomised, international, multicentre phase 2 trial. Lancet Oncol 2015;16:1651-8.

20. Voigt SL, Raman V, Jawitz OK, et al. The Role of Neoadjuvant Chemotherapy in Patients With Resectable Malignant Pleural Mesothelioma-An Institutional and National Analysis. J Natl Cancer Inst 2020;112:1118-27.

21. Marulli G, Faccioli E, Bellini A, et al. Induction chemotherapy vs post-operative adjuvant therapy for malignant pleural mesothelioma. Expert Rev Respir Med 2017;11:649-60.

22. Bölükbas S, Eberlein M, Fisseler-Eckhoff A, et al. Radical pleurectomy and chemoradiation for malignant pleural mesothelioma: the outcome of incomplete resections. Lung Cancer 2013;81:241-6.

23. Lim E, Darlison L, Edwards J, et al. Mesothelioma and Radical Surgery 2 (MARS 2): protocol for a multicentre randomised trial comparing (extended) pleurectomy decortication versus no (extended) pleurectomy decortication for patients with malignant pleural mesothelioma. BMJ Open 2020;10:e038892.

24. Hassan R, Blumenschein GR Jr, Moore KN, et al. First-in-Human, Multicenter, Phase I Dose-Escalation and Expansion Study of Anti-Mesothelin AntibodyDrug Conjugate Anetumab Ravtansine in Advanced or Metastatic Solid Tumors. J Clin Oncol 2020;38:1824-35.

25. Guazzelli A, Meysami P, Bakker E, et al. BAP1 Status Determines the Sensitivity of Malignant Mesothelioma Cells to Gemcitabine Treatment. Int J Mol Sci 2019;20:429.

26. Ladanyi M, Sanchez Vega F, Zauderer M. Loss of BAP1 as a candidate predictive biomarker for immunotherapy of mesothelioma. Genome Med 2019;11:18.

27. Szlosarek PW, Steele JP, Nolan L, et al. Arginine 
Deprivation With Pegylated Arginine Deiminase in Patients With Argininosuccinate Synthetase 1-Deficient Malignant Pleural Mesothelioma: A Randomized Clinical Trial. JAMA Oncol 2017;3:58-66.

28. Beddowes E, Spicer J, Chan PY, et al. Phase 1 DoseEscalation Study of Pegylated Arginine Deiminase, Cisplatin, and Pemetrexed in Patients With Argininosuccinate Synthetase 1-Deficient Thoracic

doi: $10.21037 /$ asj-21-55

Cite this article as: Edelman MJ. Role of induction and adjuvant systemic treatment in mesothelioma: narrative review. AME Surg J 2022;2:15.
Cancers. J Clin Oncol 2017;35:1778-85.

29. Ettinger DS, Wood DE, Akerley W, et al. NCCN Guidelines Insights: Malignant Pleural Mesothelioma, Version 3.2016. J Natl Compr Canc Netw 2016;14:825-36.

30. Kindler HL, Ismaila N, Armato SG 3rd, et al. Treatment of Malignant Pleural Mesothelioma: American Society of Clinical Oncology Clinical Practice Guideline. J Clin Oncol 2018;36:1343-73. 